\title{
REGULAR COVERING SPACE IN DIGITAL COVERING THEORY AND ITS APPLICATIONS
}

\author{
SANG-EON HAN
}

\begin{abstract}
As a survey-type article, the paper reviews some results on a regular covering space in digital covering theory. The recent paper [10](see also [12]) established the notion of regular covering space in digital covering theory and studied its various properties. Besides, the papers $[14,16]$ developed a discrete Deck's transformation group of a digital covering. In this paper we study further their properties. By using these properties, we can classify digital covering spaces. Finally, the paper proposes an open problem.
\end{abstract}

\section{Introduction}

Let $\mathbf{N}, \mathbf{Z}$, and $\mathbf{R}$ denote the sets of natural numbers, integers, and real numbers, respectively. Let $\mathbf{Z}^{n}$ denote the set of points in the Euclidean $n$-dimensional space with integer coordinates. Useful tools from algebraic topology and geometric topology for studying digital topological properties of a (binary) digital space include a digital covering space, the (digital) $k$-fundamental group, a digital $k$-surface, and so forth. These have been studied in papers including $[1,2,6,7,8,9,10,11,12,13$, $14,15,16,17,18,19,20,21,22,25,26,31]$. Recently, motivated by both a regular covering space and a Deck's transformation group in algebraic topology [32], their digital versions in digital covering theory have been developed [10, 12, 14, 16], which play important roles in classifying digital covering spaces. The paper studies further their properties.

The paper is organized as follows. Section 2 provides some basic notions. Section 3 investigates some properties of a regular covering space in digital covering theory in relation to a calculation of an automorphism group (or discrete Deck's transformation group) of a digital

Received June 24, 2009. Accepted September 7, 2009.

2000 Mathematics Subject Classification: 55Q70,52Cxx,55P15,68R10,68U05.

Key words and phrases: digital space, digital isomorphism, strong $k$ deformation retract, regular covering space, digital covering space, simply $k$ connected, discrete Deck's transformation group, automorphism group. 
covering space. Finally, Section 4 concludes the paper with a concluding remark and a further work, and proposes an open problem relating to an automorphism group of a digital covering which cannot satisfy a radius 2 - $\left(k_{0}, k_{1}\right)$-isomorphism.

\section{Preliminaries}

Motivated by the $k$-adjacency relations of $2 D$ and $3 D$ digital spaces in $[28,30]$, the $k$-adjacency relations of a multi-dimensional digital space in $\mathbf{Z}^{n}$, induced from the following operator, have been established [4] (see also [7]):

For a natural number $m$ with $1 \leq m \leq n$, two distinct points

$$
p=\left(p_{1}, p_{2}, \cdots, p_{n}\right), q=\left(q_{1}, q_{2}, \cdots, q_{n}\right) \in \mathbf{Z}^{n},
$$

are $k(m, n)$ - (briefly, $k_{m^{-}}$or $k^{-}$) adjacent if

- there are at most $m$ indices $i$ such that $\left|p_{i}-q_{i}\right|=1$ and

- for all other indices $i$ such that $\left|p_{i}-q_{i}\right| \neq 1, p_{i}=q_{i}$.

Then, we observe that the $k(m, n)$ (briefly $k$ or $k_{m}$ )-adjacency relations of $\mathbf{Z}^{n}$ are determined according to the two numbers $m, n \in \mathbf{N}$ [4] (see also [7, 22]), as follows.

Proposition 2.1. [18] $k:=k(m, n)=\sum_{i=n-m}^{n-1} 2^{n-i} C_{i}^{n}$, where $C_{i}^{n}=$ $\frac{n !}{(n-i) ! i !}$.

The current $k$-adjacency relations of $\mathbf{Z}^{n}$ is often used in studying digital topological properties of a multi-dimensional digital space in $\mathbf{Z}^{n}$, $n \in \mathbf{N}$.

In general, a pair $(X, k)$ is assumed to be a (binary) digital space with $k$-adjacency in a quadruple $\left(\mathbf{Z}^{n}, k, \bar{k}, X\right)$, where $(k, \bar{k}) \in\{(k, 2 n),(2 n$, $\left.\left.3^{n}-1\right)\right\}, k$ represents an adjacency relation for $X$, and $\bar{k}$ represents an adjacency relation for $\mathbf{Z}^{n}-X[28]$. Furthermore, owing to the digital $k$-connectivity paradox in [28], we remind $k \neq \bar{k}$ except for the case $(\mathbf{Z}, 2,2, X)$. For $\{a, b\} \subset \mathbf{Z}$ with $a \leq b,[a, b]_{\mathbf{Z}}=\{a \leq n \leq b \mid n \in \mathbf{Z}\}$ is considered in $(\mathbf{Z}, 2,2,[a, b] \mathbf{Z})[1]$. But in this paper we are not concerned with $\bar{k}$-adjacency between two points in $\mathbf{Z}^{n}-X$.

We say that two subsets $(A, k)$ and $(B, k)$ of $(X, k)$ are $k$-adjacent to each other if $A \cap B=\emptyset$ and there are points $a \in A$ and $b \in B$ such that $a$ and $b$ are $k$-adjacent to each other [28]. We say that a set $X \subset \mathbf{Z}^{n}$ is $k$-connected if it is not a union of two disjoint non-empty sets that are not $k$-adjacent to each other [28]. If a digital space $(X, k)$ is $k$-connected, then for any two points $x, y \in X$ there is a $k$-path from $x$ to $y$ in $X$ [28]. 
For an adjacency relation $k$ of $\mathbf{Z}^{n}$, a simple $k$-path with $l$ elements in $\mathbf{Z}^{n}$ is assumed to be a $k$-sequence $\left(x_{i}\right)_{i \in[0, l]_{\mathbf{Z}}} \subset \mathbf{Z}^{n}$ such that $x_{i}$ and $x_{j}$ are $k$-adjacent and further, $x_{0}=x$ and $x_{l}=y$ if and only if either $j=i+1$ or $i=j+1$ [28]. We say that the length of the simple $k$-path, denoted by $l_{k}(x, y)$, is the number $l$. A simple closed $k$-curve with $l$ elements in $\mathbf{Z}^{n}$ is a sequence $\left(x_{i}\right)_{i \in[0, l-1]_{\mathbf{Z}}}$ derived from a simple $k$-curve $\left(x_{i}\right)_{i \in[0, l]_{\mathbf{Z}}}$ with $x_{0}=x_{l}$, where $x_{i}$ and $x_{j}$ are $k$-adjacent if and only if $j=i+1(\bmod l)$ or $i=j+1(\bmod l)[28]$. Let $S C_{k}^{n, l}$ denote a simple closed $k$-curve with $l$ elements in $\mathbf{Z}^{n}[7]$.

In order to study digital continuity and various properties of digital geometry including both a digital $k$-curve and a digital $k$-surface $[9,11]$, we have used the following digital $k$-neighborhood of a point $x \in X$ with radius $\varepsilon \in \mathbf{N}$.

Definition 1. [4] (see also [7]) For a digital space $(X, k)$ in $\mathbf{Z}^{n}$, the digital $k$-neighborhood of $x_{0} \in X$ with radius $\varepsilon$ is defined in $X$ to be the following subset of $X$

$$
N_{k}\left(x_{0}, \varepsilon\right)=\left\{x \in X \mid l_{k}\left(x_{0}, x\right) \leq \varepsilon\right\} \cup\left\{x_{0}\right\},
$$

where $l_{k}\left(x_{0}, x\right)$ is the length of a shortest simple $k$-path from $x_{0}$ to $x$ and $\varepsilon \in \mathbf{N}$.

Motivated by both the digital continuity in [30] and the $\left(k_{0}, k_{1}\right)$ continuity in [1], the following notion of digital continuity in a form has been often used for the study of a digital space in $\mathbf{Z}^{n}, n \in \mathbf{N}$.

Proposition 2.2. [4] (see also [7]) Let $\left(X, k_{0}\right)$ and $\left(Y, k_{1}\right)$ be digital spaces in $\mathbf{Z}^{n_{0}}$ and $\mathbf{Z}^{n_{1}}$, respectively. A function $f: X \rightarrow Y$ is $\left(k_{0}, k_{1}\right)$ continuous if and only if for every $x \in X, \varepsilon \in \mathbf{N}$, and $N_{k_{1}}(f(x), \varepsilon) \subset Y$, there is $\delta \in \mathbf{N}$ such that the corresponding $N_{k_{0}}(x, \delta) \subset X$ satisfies $f\left(N_{k_{0}}(x, \delta)\right) \subset N_{k_{1}}(f(x), \varepsilon)$.

Indeed, the current $\left(k_{0}, k_{1}\right)$-continuity of $f:\left(X, k_{0}\right) \rightarrow\left(Y, k_{1}\right)$ at a point $x \in X$ is equivalent to the following statement because every point $x$ of a digital space $(X, k)$ always has an $N_{k}(x, 1)$ in $(X, k)$.

Proposition 2.3. [16] Let $\left(X, k_{0}\right)$ and $\left(Y, k_{1}\right)$ be digital spaces in $\mathbf{Z}^{n_{0}}$ and $\mathbf{Z}^{n_{1}}$, respectively. A function $f: X \rightarrow Y$ is $\left(k_{0}, k_{1}\right)$-continuous if and only if for every $x \in X f\left(N_{k_{0}}(x, 1)\right) \subset N_{k_{1}}(f(x), 1)$.

Unlike the pasting property of classical continuity in topology, $\left(k_{0}, k_{1}\right)$ continuity has some intrinsic features [27]: Digital $\left(k_{0}, k_{1}\right)$-continuity has the almost pasting property instead of the pasting property of classical topology. 
Since a digital space $(X, k)$ can be recognized to be a digital $k$-graph, we may use the term a $\left(k_{0}, k_{1}\right)$-isomorphism as in $[2,8]$ rather than a $\left(k_{0}, k_{1}\right)$-homeomorphism as in [1]:

Definition 2. [8] For two digital spaces $\left(X, k_{0}\right)$ in $\mathbf{Z}^{n_{0}}$ and $\left(Y, k_{1}\right)$ in $\mathbf{Z}^{n_{1}}$, a map $h: X \rightarrow Y$ is called a $\left(k_{0}, k_{1}\right)$-isomorphism if $h$ is a $\left(k_{0}, k_{1}\right)$ continuous bijection and further, $h^{-1}: Y \rightarrow X$ is $\left(k_{1}, k_{0}\right)$-continuous. Then we use the notation $X \approx_{\left(k_{0}, k_{1}\right)} Y$. If $n_{0}=n_{1}$ and $k_{0}=k_{1}$, then we call it a $k_{0}$-isomorphism and use the notation $X \approx_{k_{0}} Y$.

For a digital space $(X, k)$ and $A \subset X,(X, A)$ is called a digital space pair with $k$-adjacency [9]. Furthermore, if $A$ is a singleton set $\left\{x_{0}\right\}$, then $\left(X, x_{0}\right)$ is called a pointed digital space [28]. Motivated by the pointed digital homotopy in [1], the following notion of relative $k$-homotopy to a subset $A \subset X$ is often used in studying a $k$-homotopic thinning and a strong $k$-deformation retract of a digital space $(X, k)$ in $\mathbf{Z}^{n}$.

Definition 3. [9] (see also $[10,14])$ Let $\left((X, A), k_{0}\right)$ and $\left(Y, k_{1}\right)$ be a digital space pair and a digital space, respectively. Let $f, g: X \rightarrow Y$ be $\left(k_{0}, k_{1}\right)$-continuous functions. Suppose there exist $m \in \mathbf{N}$ and a function $F: X \times[0, m]_{\mathbf{Z}} \rightarrow Y$ such that

- for all $x \in X, F(x, 0)=f(x)$ and $F(x, m)=g(x)$;

- for all $x \in X$, the induced function $F_{x}:[0, m]_{\mathbf{Z}} \rightarrow Y$ given by

$F_{x}(t)=F(x, t)$ for all $t \in[0, m]_{\mathbf{Z}}$ is $\left(2, k_{1}\right)$-continuous;

- for all $t \in[0, m]_{\mathbf{z}}$, the induced function $F_{t}: X \rightarrow Y$ given by $F_{t}(x)=$ $F(x, t)$ for all $x \in X$ is $\left(k_{0}, k_{1}\right)$-continuous.

Then we say that $F$ is a $\left(k_{0}, k_{1}\right)$-homotopy between $f$ and $g$ [1].

- Furthermore, for all $t \in[0, m]_{\mathbf{Z}}$, then suppose the induced map $F_{t}$ on $A$ is a constant which is the prescribed function from $A$ to $Y$. In other words, $F_{t}(x)=f(x)=g(x)$ for all $x \in A$ and for all $t \in[0, m]_{\mathbf{Z}}$.

Then we call $F$ a $\left(k_{0}, k_{1}\right)$-homotopy relative to $A$ between $f$ and $g$, and we say $f$ and $g$ are $\left(k_{0}, k_{1}\right)$-homotopic relative to $A$ in $Y$. Then we use the notation $f \simeq_{\left(k_{0}, k_{1}\right) \text { relA }} g$.

In Definition 3, if $A=\left\{x_{0}\right\} \subset X$, then we say that $F$ is a pointed $\left(k_{0}, k_{1}\right)$-homotopy at $\left\{x_{0}\right\}[1]$. When $f$ and $g$ are pointed $\left(k_{0}, k_{1}\right)$ homotopic in $Y$, we use the notation $f \simeq_{\left(k_{0}, k_{1}\right)} g$. In addition, if $k_{0}=k_{1}$ and $n_{0}=n_{1}$, then we say that $f$ and $g$ are pointed $k_{0}$-homotopic in $Y$ and use the notation $f \simeq_{k_{0}} g$ and $f \in[g]$ which means the $k_{0}$-homotopy class of $g$. If, for some $x_{0} \in X, 1_{X}$ is $k$-homotopic to the constant map with space $x_{0}$ relative to $\left\{x_{0}\right\}$, then we say that $\left(X, x_{0}\right)$ is pointed $k$-contractible [1]. Indeed, the notion of $k$-contractibility is slightly different from the contractibility in Euclidean topology [1] (see also [7]). 
Motivated by Khalimsky's (digital) $k$-fundamental group in [25], the paper [1] establishes the $k$-fundamental group by using the trivial extension in [1] and the Khalimsky operation in [25]: Precisely, if $m_{f} \leq m_{f^{\prime}}$, we can obtain a trivial extension of a loop $f:\left[0, m_{f}\right]_{\mathbf{Z}} \rightarrow X$ to a loop $f^{\prime}:\left[0, m_{f^{\prime}}\right] \mathbf{z} \rightarrow X$ via

$$
f^{\prime}(t)=\left\{\begin{array}{r}
f(t) \quad \text { if } \quad 0 \leq t \leq m_{f} \\
f\left(m_{f}\right) \quad \text { if } \quad m_{f} \leq t \leq m_{f^{\prime}} .
\end{array}\right.
$$

Let us now recall the Khalimsky operation in [25]: Let $F^{k}\left(X, x_{0}\right)=$ $\left\{f \mid f\right.$ is a $k$ - loop based at $\left.x_{0}\right\}$. For members $f:\left[0, m_{f}\right]_{\mathbf{Z}} \rightarrow X$ and $g:\left[0, m_{g}\right] \mathbf{z} \rightarrow X$ of $F^{k}\left(X, x_{0}\right)$, the Khalimsky operation $f * g:$ $\left[0, m_{f}+m_{g}\right]_{\mathbf{Z}} \rightarrow X$ is defined by

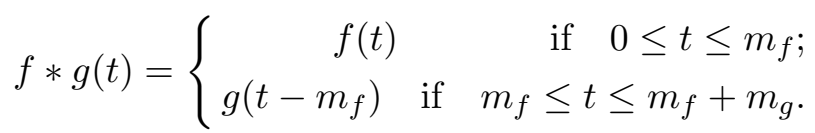

For a digital space $(X, k)$, consider a $k$-loop $f$ with base point $x_{0}$, we denote $[f]_{X}$ (briefly, $[f]$ ) the $k$-homotopy class of $f$ in $X$. Then, for a $k$-loop $f_{1}$ with the same base point $x_{0} \in X, f_{0} \in[f]$ means that the two $k$-loops $f$ and $f_{0}$ have trivial extensions that can be joined by a $k$-homotopy keeping the end point fixed [7] (see also [2]). Furthermore, if $f_{1}, f_{2}, g_{1}, g_{2} \in F^{k}\left(X, x_{0}\right), f_{1} \in\left[f_{2}\right]$, and $g_{1} \in\left[g_{2}\right]$, then $f_{1} * g_{1} \in$ $\left[f_{2} * g_{2}\right]$, i.e., $\left[f_{1} * g_{1}\right]=\left[f_{2} * g_{2}\right][1,25]$. Then, we use the notation $\pi^{k}\left(X, x_{0}\right)=\left\{[f] \mid f \in F^{k}\left(X, x_{0}\right)\right\}$ which is a group [1] with the operation $[f] \cdot[g]=[f * g]$ and called the (digital) $k$-fundamental group of $\left(X, x_{0}\right)$ [1]. The base point of $\pi^{k}\left(X, x_{0}\right)$ should be taken as a point $x_{0} \in X$ which cannot be deleted by a strong $k$-deformation retract $[13,14,23]$. If $X$ is pointed $k$-contractible, then $\pi^{k}\left(X, x_{0}\right)$ is proved trivial [1].

Let $((X, A), k)$ be a digital space pair with $k$-adjacency. A map $f:\left((X, A), k_{0}\right) \rightarrow\left((Y, B), k_{1}\right)$ is called $\left(k_{0}, k_{1}\right)$-continuous if $f$ is $\left(k_{0}, k_{1}\right)$ continuous and $f(A) \subset B$ [9]. If $A=\{a\}, B=\{b\}$, we write $(X, A)=$ $(X, a),(Y, B)=(Y, b)$, and we say that $f$ is a pointed $\left(k_{0}, k_{1}\right)$-continuous map [28]. A $\left(k_{0}, k_{1}\right)$-continuous map $f:\left(\left(X, x_{0}\right), k_{0}\right) \rightarrow\left(\left(Y, y_{0}\right), k_{1}\right)$ induces a group homomorphism $f_{*}: \pi^{k_{0}}\left(X, x_{0}\right) \rightarrow \pi^{k_{1}}\left(Y, y_{0}\right)$ given by $f_{*}([\alpha])=[f \circ \alpha]$, where $[\alpha] \in \pi^{k_{0}}\left(X, x_{0}\right)[1]$. In addition, a $\left(k_{0}, k_{1}\right)$ isomorphism $\phi:\left(\left(X, x_{0}\right), k_{0}\right) \rightarrow\left(\left(Y, y_{0}\right), k_{1}\right)$ induces a group isomorphism $\phi_{*}: \pi^{k_{0}}\left(X, x_{0}\right) \rightarrow \pi^{k_{1}}\left(Y, y_{0}\right)[1]$.

The following notion has been often used in digital $k$-homotopy theory and digital covering theory. 
Definition 4. [7] A pointed $k$-connected digital space $\left(X, x_{0}\right)$ is called simply $k$-connected if $\pi^{k}\left(X, x_{0}\right)$ is a trivial group.

\section{Some Properties of a Regular Covering Space in Digital Covering Theory}

For the purpose of calculating $\pi^{k}\left(X, x_{0}\right)$ and classifying digital spaces, we have often used some properties of a $\left(k_{0}, k_{1}\right)$-covering [6, 7]. In digital covering theory each digital space $(X, k)$ is assumed to be $k$-connected.

Definition 5. [7] (see also $[10,16])$ Let $\left(E, k_{0}\right)$ and $\left(B, k_{1}\right)$ be digital spaces in $\mathbf{Z}^{n_{0}}$ and $\mathbf{Z}^{n_{1}}$, respectively and $p: E \rightarrow B$ a $\left(k_{0}, k_{1}\right)$-continuous surjection. Suppose, for any $b \in B$, there exists $\varepsilon \in \mathbf{N}$ such that

(1) for some index set $M, p^{-1}\left(N_{k_{1}}(b, \varepsilon)\right)=\cup_{i \in M} N_{k_{0}}\left(e_{i}, \varepsilon\right)$ with $e_{i} \in$ $p^{-1}(b)$;

(2) if $i, j \in M$ and $i \neq j$, then $N_{k_{0}}\left(e_{i}, \varepsilon\right) \cap N_{k_{0}}\left(e_{j}, \varepsilon\right)$ is an empty set; and

(3) the restriction map $p$ on $N_{k_{0}}\left(e_{i}, \varepsilon\right)$ is a $\left(k_{0}, k_{1}\right)$-isomorphism for all $i \in M$.

Then, the map $p$ is called a $\left(k_{0}, k_{1}\right)$-covering map and $(E, p, B)$ is said to be a $\left(k_{0}, k_{1}\right)$-covering.

The $k_{1}$-neighborhood $N_{k_{1}}(b, \varepsilon)$ in Definition 5 is called an elementary $k_{1}$-neighborhood of $b$ with some radius $\varepsilon$ and further, $E$ is called a $\left(k_{0}, k_{1}\right)$ covering space of $p$. For instance a $(2, k)$-covering map, let $\left(\mathbf{Z}, p, S C_{k}^{n, l}:=\right.$ $\left.\left(c_{t}\right)_{t \in[0, l-1]_{\mathbf{Z}}}\right)$ is a $(2, k)$-covering map [7], where $p(t)=c_{t(\bmod l)}, t \in \mathbf{Z}$.

For any two points $b_{0}, b_{1} \in B$ of a $\left(k_{0}, k_{1}\right)$-covering $\left(E, e_{0}\right), p,\left(B, b_{0}\right)$, since the cardinalities of $p\left(b_{0}\right)$ and $p\left(b_{1}\right)$ are equal [23], we can obtain the following:

Definition 6. [10] (see also [16]) We say that a $\left(k_{0}, k_{1}\right)$-covering map $p:\left(E, e_{0}\right) \rightarrow\left(B, b_{0}\right)$ is an $n$-fold $\left(k_{0}, k_{1}\right)$-covering map if the cardinality of the index set $M$ is $n$.

Namely, Definition 6 can be represented as follows. For a $\left(k_{0}, k_{1}\right)$ covering map $p:\left(E, e_{0}\right) \rightarrow\left(B, b_{0}\right)$ if the set $p^{-1}\left(b_{0}\right)$ has $n$ elements (or the number $n$ can be also called the sheets of the digital covering (see [29]), then the map $p$ is called an $n$-fold $\left(k_{0}, k_{1}\right)$-covering map $[14,16]$. For instance, $\left(S C_{k}^{n, m l}:=\left(a_{t}\right)_{t \in[0, m l-1]_{\mathbf{Z}}}, p, S C_{k}^{n, l}:=\left(c_{t}\right)_{t \in[0, l-1]_{\mathbf{Z}}}\right)$ is an $m$-fold $(k, k)$-covering, where $p\left(a_{i}\right)=c_{i(\bmod l)}$.

For pointed digital spaces $\left(\left(E, e_{0}\right), k_{0}\right)$ and $\left(\left(B, b_{0}\right), k_{1}\right)$, if $p:\left(E, e_{0}\right) \rightarrow$ $\left(B, b_{0}\right)$ is a $\left(k_{0}, k_{1}\right)$-covering map such that $p\left(e_{0}\right)=b_{0}$, then the map $p$ 
is a pointed $\left(k_{0}, k_{1}\right)$-covering map [7]. Hereafter, we assume that each digital covering map is a pointed one unless stated.

Definition 7. [5] (see also $[6,17])$ For two digital spaces $\left(X, k_{0}\right)$ in $\mathbf{Z}^{n_{0}}$ and $\left(Y, k_{1}\right)$ in $\mathbf{Z}^{n_{1}}$, a $\left(k_{0}, k_{1}\right)$-continuous map $h: X \rightarrow Y$ is called a local $\left(k_{0}, k_{1}\right)$-isomorphism if for every $x \in X, h$ maps $N_{k_{0}}(x, 1) \subset X$ $\left(k_{0}, k_{1}\right)$-isomorphically onto $N_{k_{1}}(h(x), 1) \subset Y$. If $n_{0}=n_{1}$ and $k_{0}=k_{1}$, then the map $h$ is called a local $k_{0}$-isomorphism.

This local $\left(k_{0}, k_{1}\right)$-isomorphism can be strongly used in studying the investigation of the preservation of local $k_{0}$-properties of a digital space $\left(X, k_{0}\right)$ into its corresponding $k_{1}$-ones in digital geometry [17]. Since a $\left(k_{0}, k_{1}\right)$-isomorphism is equivalent to locally $\left(k_{0}, k_{1}\right)$-isomorphic bijection and a restriction map of a $\left(k_{0}, k_{1}\right)$-isomorphism is also a $\left(k_{0}, k_{1}\right)$ isomorphism [15], we obtain the following property. If $h:\left(X, k_{0}\right) \rightarrow$ $\left(Y, k_{1}\right)$ is a $\left(k_{0}, k_{1}\right)$-isomorphism, then the restriction map on $N_{k_{0}}(x, 1)$, $\left.h\right|_{N_{k_{0}}(x, 1)}: N_{k_{0}}(x, 1) \rightarrow N_{k_{1}}(h(x), 1)$, is a $\left(k_{0}, k_{1}\right)$-isomorphism [5] (see also [11]).

Remark 3.1. [10] Let $h:\left(X, k_{0}\right) \rightarrow\left(Y, k_{1}\right)$ be a $\left(k_{0}, k_{1}\right)$-isomorphism. Then, for any $X_{0} \subset X$, the restriction $\left.h\right|_{X_{0}}:\left(X_{0}, k_{0}\right) \rightarrow\left(h\left(X_{0}\right), k_{1}\right)$ is also a $\left(k_{0}, k_{1}\right)$-isomorphism [11]. Furthermore, since a $\left(k_{0}, k_{1}\right)$-isomorphism is equivalent to a local $\left(k_{0}, k_{1}\right)$-isomorphic bijection [5], we may take $\varepsilon=1$ for the $\left(k_{0}, k_{1}\right)$-covering of Definition 5 (see also [2]).

In light of Remark 3.1, even though we may take $\varepsilon=1$ in Definition 5 , in relation with Lemma 3.2 , and various digital topological properties in Section 3, we may take some $\varepsilon \in \mathbf{N}$ in Definition 5 according to the situation.

Definition 8. [6] For $n \in \mathbf{N}$, a $\left(k_{0}, k_{1}\right)$-covering $(E, p, B)$ is a radius $n$ local isomorphism if the restriction map $\left.p\right|_{N_{k_{0}}\left(e_{i}, n\right)}: N_{k_{0}}\left(e_{i}, n\right) \rightarrow$ $N_{k_{1}}(b, n)$ is a $\left(k_{0}, k_{1}\right)$-isomorphism for all $i \in M$.

Definition 9. [6] (see also [16]) A $\left(k_{0}, k_{1}\right)$-covering $(E, p, B)$ is called a radius $n$ - $\left(k_{0}, k_{1}\right)$-covering if $\varepsilon \geq n$, where the number $\varepsilon$ is the same as the $\varepsilon$ of Definition 5 .

In terms of Definitions 8 and 9 , we observe that a $\left(k_{0}, k_{1}\right)$-covering satisfying a radius $n$ local isomorphism is equivalent to a radius $n$ $\left(k_{0}, k_{1}\right)$-covering. By Remark 3.1 , we observe that a $\left(k_{0}, k_{1}\right)$-covering of Definition 5 is clearly a radius $1-\left(k_{0}, k_{1}\right)$-covering.

For three digital spaces $\left(E, k_{0}\right)$ in $\mathbf{Z}^{n_{0}},\left(B, k_{1}\right)$ in $\mathbf{Z}^{n_{1}}$, and $\left(X, k_{2}\right)$ in $\mathbf{Z}^{n_{2}}$, let $p: E \rightarrow B$ be a $\left(k_{0}, k_{1}\right)$-continuous map. For a $\left(k_{2}, k_{1}\right)$ continuous map $f:\left(X, k_{2}\right) \rightarrow\left(B, k_{1}\right)$, as the digital analogue of the 
lifting in [29], we say that a digital lifting of $f$ is a $\left(k_{2}, k_{0}\right)$-continuous map $\tilde{f}: X \rightarrow E$ such that $p \circ \tilde{f}=f[7]$. We now recall the unique digital lifting theorem in [7].

Lemma 3.2. [7] For pointed digital spaces $\left(\left(E, e_{0}\right), k_{0}\right)$ in $\mathbf{Z}^{n_{0}}$ and $\left(\left(B, b_{0}\right), k_{1}\right)$ in $\mathbf{Z}^{n_{1}}$, let $p:\left(E, e_{0}\right) \rightarrow\left(B, b_{0}\right)$ be a pointed $\left(k_{0}, k_{1}\right)$ covering map. Any $k_{1}$-path $f:[0, m]_{\mathbf{Z}} \rightarrow B$ beginning at $b_{0}$ has a unique digital lifting to a $k_{0}$-path $\tilde{f}$ in $E$ beginning at $e_{0}$.

Moreover, the following digital homotopy lifting theorem was introduced in [6], which plays an important role in studying digital covering theory.

Lemma 3.3. [6] Let $\left(\left(E, e_{0}\right), k_{0}\right)$ and $\left(\left(B, b_{0}\right), k_{1}\right)$ be pointed digital spaces. Let $p:\left(E, e_{0}\right) \rightarrow\left(B, b_{0}\right)$ be a radius $2-\left(k_{0}, k_{1}\right)$-covering map. For $k_{0}$-paths $g_{0}, g_{1}$ in $\left(E, e_{0}\right)$ that start at $e_{0}$, if there is a $k_{1}$-homotopy in $B$ from $p \circ g_{0}$ to $p \circ g_{1}$ that holds the endpoints fixed, then $g_{0}$ and $g_{1}$ have the same terminal point, and there is a $k_{0}$-homotopy in $E$ from $g_{0}$ to $g_{1}$ that holds the endpoints fixed.

Definition 10. [16] For a digital space $(B, k)$ in $\mathbf{Z}^{n}$, let $\left(E_{1}, p_{1}, B\right)$ and $\left(E_{2}, p_{2}, B\right)$ be two $\left(k_{1}, k\right)$ - and $\left(k_{2}, k\right)$-coverings, respectively. Then we say that a $\left(k_{1}, k_{2}\right)$-continuous map $\phi: E_{1} \rightarrow E_{2}$ such that $p_{2} \circ \phi=p_{1}$ is a $\left(k_{1}, k_{2}\right)$-homomorphism from $\left(E_{1}, p_{1}, B\right)$ into $\left(E_{2}, p_{2}, B\right)$.

In order to study a discrete Deck's transformation group of a digital covering map, we recall the following theorem.

Theorem 3.4. [13] Let $p_{1}:\left(E_{1}, e_{1}\right) \rightarrow\left(B, b_{0}\right)$ be a $\left(k_{1}, k_{0}\right)$-covering map and let $p_{2}:\left(E_{2}, e_{2}\right) \rightarrow\left(B, b_{0}\right)$ be a $\left(k_{2}, k_{0}\right)$-covering map. If there is a $\left(k_{1}, k_{2}\right)$-continuous map $\phi:\left(E_{1}, e_{1}\right) \rightarrow\left(E_{2}, e_{2}\right)$ such that $p_{2} \circ \phi=p_{1}$, then the map $\phi$ is a $\left(k_{1}, k_{2}\right)$-covering map.

Theorem 3.4 has been often used for the study of a discrete Deck's transformation group of a digital $\left(k_{0}, k_{1}\right)$-covering map in relation with a classification of digital covering spaces $[14,16]$.

Corollary 3.5. [20] Let $p_{1}:\left(E_{1}, e_{1}\right) \rightarrow\left(B, b_{0}\right)$ be a $\left(k_{1}, k_{0}\right)$-covering map and $p_{2}:\left(E_{2}, e_{2}\right) \rightarrow\left(B, b_{0}\right)$ a $\left(k_{2}, k_{0}\right)$-covering map. If there are both a $\left(k_{1}, k_{2}\right)$-continuous map $\phi:\left(E_{1}, e_{1}\right) \rightarrow\left(E_{2}, e_{2}\right)$ and a $\left(k_{2}, k_{1}\right)$ continuous map $\psi:\left(E_{2}, e_{2}\right) \rightarrow\left(E_{1}, e_{1}\right)$ such that $p_{2} \circ \phi=p_{1}$ and $p_{1} \circ \psi=p_{2}$, then the map $\phi$ is a $\left(k_{1}, k_{2}\right)$-isomorphism.

Definition 11. [10] (see also [12]) $A\left(k_{0}, k_{1}\right)$-covering $\left(\left(E, e_{0}\right), p,\left(B, b_{0}\right)\right)$ is called a regular $\left(k_{0}, k_{1}\right)$-covering if $p_{*} \pi^{k_{0}}\left(E, e_{0}\right)$ is a normal subgroup of $\pi^{k_{1}}\left(B, b_{0}\right)$. 
A discrete Deck's transformation group of a digital $\left(k_{0}, k_{1}\right)$-covering has some advantages which lie so convenient and efficient for calculating the digital fundamental group of some digital spaces and classifying digital spaces $[14,16]$. As the digital version of Deck's transformation group of a covering map in algebraic topology [32], we obtain the following:

Definition 12. [16] (see also $[14,26])$ Consider a $\left(k_{0}, k_{1}\right)$-covering map $p:\left(\left(E, e_{0}\right), k_{0}\right) \rightarrow\left(\left(B, b_{0}\right), k_{1}\right)$. A self $k_{0}$-isomorphism of the $\left(k_{0}, k_{1}\right)$-covering map $p$, denoted by $h:\left(E, e_{0}\right) \rightarrow\left(E, e_{0}\right)$, is called a $k_{0}$-covering transformation, a discrete Deck's transformation [26], or a discrete automorphism of a digital covering map $p$ if $p=p \circ h$, where - means the composition. The set of the discrete automorphisms of a digital covering map with composition operation is obviously a group which is denoted by $\operatorname{Aut}\left(\left(E, e_{0}\right) \mid\left(B, b_{0}\right)\right)$.

For a $\left(k_{0}, k_{1}\right)$-covering map $p:\left(\left(E, e_{0}\right), k_{0}\right) \rightarrow\left(\left(B, b_{0}\right), k_{1}\right)$ in order to study a relation between $p^{-1}\left(b_{0}\right)$ and a coset $\pi^{k_{1}}\left(B, b_{0}\right) / p_{*} \pi^{k_{0}}\left(E, e_{0}\right)$, we often use the following property.

Lemma 3.6. [29] Let $E$ be a set and $G$ a group. If $E \times G \rightarrow E$ is a transitive action, then $E$ is isomorphic to the factor group $G / G_{x_{0}}$, where $G_{x_{0}}:=\left\{g \in G \mid g\left(x_{0}\right)=x_{0}\right\}$ called the isotropy subgroup of $G$.

Using Massey's program [29], we obtain the following:

Theorem 3.7. Let $\left(\left(E, e_{0}\right), p,\left(B, b_{0}\right)\right)$ be a pointed radius $2-\left(k_{0}, k_{1}\right)$ covering and $\left(E, k_{0}\right) k_{0}$-connected. Then we obtain the following. (1) $\pi^{k_{1}}\left(B, b_{0}\right)$ operates transitively on $p^{-1}\left(b_{0}\right)$ on the right [12].

(2) The right $\pi^{k_{1}}\left(B, b_{0}\right)$-space $p^{-1}\left(b_{0}\right)$ is bijective to $\pi^{k_{1}}\left(B, b_{0}\right) / p_{*} \pi^{k_{0}}$ $\left(E, e_{0}\right)$.

Proof: (1) Consider $e \in p^{-1}\left(b_{0}\right)$ and $\alpha \in \pi^{k_{1}}\left(B, b_{0}\right)$. Take a $k_{1}$-path $f:\left[0, m_{f}\right] \mathbf{z} \rightarrow\left(B, b_{0}\right)$ such that $[f]=\alpha, f(0)=b_{0}=f\left(m_{f}\right)$ Then, by Lemma 3.3, the map $f$ is well-defined . By Lemma 3.2, there is the unique $k_{0}$-path $\tilde{f}:\left[0, m_{f}\right]_{\mathbf{Z}} \rightarrow\left(E, e_{0}\right)$ such that $p_{*}([\tilde{f}])=[f]$ and $\tilde{f}(0)=e$. Precisely, define

$$
p^{-1}\left(b_{0}\right) \times \pi^{k_{1}}\left(B, b_{0}\right) \rightarrow p^{-1}\left(b_{0}\right)
$$

by

$$
(e, \alpha) \rightarrow e \cdot \alpha=\tilde{f}\left(m_{f}\right)
$$

and by Lemma 3.3 this action is well-defined because this process does not depend on the choice of the map $f$, where $[f]=\alpha$. Then, for $e \in p^{-1}\left(b_{0}\right)$ we clearly observe [12]

$$
(e, 1)=e \quad \text { and } \quad((e, \alpha), \beta)=(e, \alpha \cdot \beta),
$$


where $\alpha, \beta \in \pi^{k_{1}}\left(B, b_{0}\right)$ and 1 is the identity element. This implies $p^{-1}\left(b_{0}\right)$ admits $\pi^{k_{1}}\left(B, b_{0}\right)$ as a group of operator (or permutation). The property (3.1) completes the proof.

(2) Take $\alpha \in p_{*} \pi^{k_{0}}\left(E, e_{0}\right)$. Then there exists $[\tilde{f}] \in \pi^{k_{0}}\left(E, e_{0}\right)$ such that $\alpha=[p \tilde{f}]$. Then we obtain $e_{0} \cdot \alpha=\tilde{f}\left(m_{\tilde{f}}\right)=e_{0}$ and finally, $\alpha \in$ $\left(\pi^{k_{1}}\left(B, b_{0}\right)\right)_{e_{0}}$.

If $\beta \in\left(\pi^{k_{1}}\left(B, b_{0}\right)\right)_{e_{0}}$ with $\beta=[g]$ and $g:\left[0, m_{g}\right]_{\mathbf{Z}} \rightarrow\left(B, b_{0}\right)$ represents $\beta$, i.e., $e_{0} \cdot \beta=\tilde{g}\left(m_{g}\right)=e_{0}$ which is well-defined by Lemma 3.3. Thus $[\tilde{g}] \in \pi^{k_{0}}\left(E, e_{0}\right)$ and $[p \tilde{g}]=p_{*}[\tilde{g}] \in p_{*} \pi^{k_{0}}\left(E, e_{0}\right)$. Consequently, we obtain $p_{*} \pi^{k_{0}}\left(E, e_{0}\right)=\left(\pi^{k_{1}}\left(B, b_{0}\right)\right)_{e_{0}}$. Thus, by Lemma 3.6, $p^{-1}\left(b_{0}\right)$ is proved to be bijective to the factor group $\pi^{k_{1}}\left(B, b_{0}\right) / p_{*} \pi^{k_{0}}\left(E, e_{0}\right)$.

Remark 3.8. Let us remind that, by Theorem 3.7(2), the group $p^{-1}\left(b_{0}\right)$ in $[12,18,20]$ related to the assertion of Theorem 3.7(2) is clearly bijective to the factor group $\pi^{k_{1}}\left(B, b_{0}\right) / p_{*} \pi^{k_{0}}\left(E, e_{0}\right)$ as a right $\pi^{k_{1}}\left(B, b_{0}\right)$-space, and the papers $[12,18]$ tells further the case $p^{-1}\left(b_{0}\right)$ has some group structure such as $\left(p^{-1}\left(b_{0}\right),+\right)$ derived from the given digital coverings such as $\left(\mathbf{Z}, p, S C_{k}^{n, l}\right)$ and $\left(\mathbf{Z} \times \mathbf{Z}, p_{1} \times p_{2}, S C_{k_{1}}^{n_{1}, l_{1}} \times\right.$ $\left.S C_{k_{2}}^{n_{2}, l_{2}}\right)$, where the $k$-adjacency of $\left(S C_{k_{1}}^{n_{1}, l_{1}} \times S C_{k_{2}}^{n_{2}, l_{2}}, k\right)$ has the $L_{S^{-}}$ or $L_{C}$-property relative to $S C_{k_{i}}^{n_{i}, l_{i}}, i \in\{1,2\}$ (see $[10,12,18,20]$ ). Of course, in this case we clearly observe that the given digital covering map $p$ should be regular so that $\pi^{k_{1}}\left(B, b_{0}\right) / p_{*} \pi^{k_{0}}\left(E, e_{0}\right)$ is clearly isomorphic to $N\left(p_{*} \pi^{k_{0}}\left(E, e_{0}\right)\right) / p_{*} \pi^{k_{0}}\left(E, e_{0}\right)$.

Lemma 3.9. [3] Let $\left(\left(E, e_{0}\right), p,\left(B, b_{0}\right)\right)$ be a pointed radius $2-\left(k_{0}, k_{1}\right)$ covering and $\left(E, k_{0}\right) k_{0}$-connected. For any $\phi \in A u t(E \mid B)$, any $e \in$ $p^{-1}\left(b_{0}\right)$ and any $\alpha \in \pi^{k_{1}}\left(B, b_{0}\right)$ we obtain $\phi(e \cdot \alpha)=\phi(e) \cdot \alpha$.

Proof: Take $\alpha=[f]$, where $f:\left[0, m_{f}\right] \mathbf{z} \rightarrow\left(B, b_{0}\right)$ represents $\alpha$. Consider the digital lifting $\tilde{f}$ of $f$ such that $\phi \tilde{f}:\left[0, m_{f}\right]_{\mathbf{Z}} \rightarrow E$ such that $\phi \tilde{f}(0)=\phi(e)$ and $\phi \tilde{f}\left(m_{f}\right)=\phi(e \cdot \alpha)$ and $p \phi \tilde{f}=p \tilde{f}=f$. Thus $\phi \tilde{f}$ is a digital lifting of $f$. Therefore, we obtain $\phi(e) \cdot \alpha=\phi \tilde{f}\left(m_{f}\right)=\phi(e \cdot \alpha)$.

Theorem 3.10. Let $\left(\left(E, e_{0}\right), p,\left(B, b_{0}\right)\right)$ be a pointed radius $2-\left(k_{0}, k_{1}\right)$ covering and $\left(E, k_{0}\right) k_{0}$-connected. Then $\operatorname{Aut}(E \mid B)$ is isomorphic to $\operatorname{Aut}\left(p^{-1}\left(b_{0}\right)\right)$, where $\operatorname{Aut}\left(p^{-1}\left(b_{0}\right)\right)$ is considered as a right $\pi^{k_{1}}\left(B, b_{0}\right)$ space. 
Proof: Define the map

$$
H: A u t(E \mid B) \rightarrow A u t\left(p^{-1}\left(b_{0}\right)\right)
$$

given by

$$
\phi \rightarrow H(\phi):=\left.\phi\right|_{p^{-1}\left(b_{0}\right)}: p^{-1}\left(b_{0}\right) \rightarrow p^{-1}\left(b_{0}\right) .
$$

First, we can prove that $H$ is injective, as follows. Assume $\left.\phi_{1}\right|_{p^{-1}\left(b_{0}\right)}=$ $\left.\phi_{2}\right|_{p^{-1}\left(b_{0}\right)}$, then we obtain $\phi_{1}\left(e_{0}\right)=\phi_{2}\left(e_{0}\right)$. Thus, $\phi_{1}=\phi_{2}$.

Second, we can prove that $H$ is surjective, as follows. Take any $f \in$ $\operatorname{Aut}\left(p^{-1}\left(b_{0}\right)\right)$. Then $f: p^{-1}\left(b_{0}\right) \rightarrow p^{-1}\left(b_{0}\right)$ given by $e_{0} \rightarrow e_{1}=f\left(e_{0}\right)$. Thus, $\left(\pi^{k_{1}}\left(B, b_{0}\right)\right)_{e_{0}}=\left(\pi^{k_{1}}\left(B, b_{0}\right)\right)_{e_{1}}$ with $\pi^{k_{1}}\left(B, b_{0}\right)_{e_{0}}=p_{*} \pi^{k_{0}}\left(E, e_{0}\right)$ and $\pi^{k_{1}}\left(B, b_{0}\right)_{e_{1}}=p_{*} \pi^{k_{0}}\left(E, e_{1}\right)$. Therefore, there exists $\left(k_{0}, k_{0}\right)$-isomorphism $\phi: E \rightarrow E$ such that $\phi\left(e_{0}\right)=e_{1}$. Namely, $\phi \in A u t(E \mid B)$ such that $\phi\left(e_{0}\right)=e_{1}$. Consequently, we obtain $H(\phi):=\left.\phi\right|_{p^{-1}\left(b_{0}\right)}=f$.

Corollary 3.11. [23] Let $\left(\left(E, e_{0}\right), p,\left(B, b_{0}\right)\right)$ be a pointed radius 2$\left(k_{0}, k_{1}\right)$-covering and $\left(E, k_{0}\right) k_{0}$-connected. Then we obtain $\operatorname{Aut}(E \mid B) \simeq N\left(p_{*} \pi^{k_{0}}\left(E, e_{0}\right)\right) / p_{*} \pi^{k_{0}}\left(E, e_{0}\right)$.

Corollary 3.12. [23] Let $\left(\left(E, e_{0}\right), p,\left(B, b_{0}\right)\right)$ be a pointed radius 2$\left(k_{0}, k_{1}\right)$-covering which is regular and $\left(E, k_{0}\right) k_{0}$-connected. Then we obtain

$\operatorname{Aut}(E \mid B) \simeq \operatorname{Aut}\left(p^{-1}\left(b_{0}\right)\right) \simeq \pi^{k_{1}}\left(B, b_{0}\right) / p_{*} \pi^{k_{0}}\left(E, e_{0}\right)$.

Theorem 3.13. Let $\left(\left(E, e_{0}\right), p,\left(B, b_{0}\right)\right)$ be a pointed radius $2-\left(k_{0}, k_{1}\right)$ covering and $\left(E, k_{0}\right) k_{0}$-connected. $\left(\left(E, e_{0}\right), p,\left(B, b_{0}\right)\right)$ is regular if and only if $p_{*} \pi^{k_{0}}\left(E, e_{0}\right)=p_{*} \pi^{k_{0}}\left(E, e_{1}\right)$, where $p\left(e_{0}\right)=p\left(e_{1}\right)=b_{0}$

Proof: If $\left(\left(E, e_{0}\right), p,\left(B, b_{0}\right)\right)$ is regular, then let $\tilde{w}:[0, m]_{\mathbf{Z}} \rightarrow E$ be a $k_{0}$-loop at $e_{0}$. Then $\tilde{w}$ is a digital closed lifting of $p(\tilde{w})$. Thus, there is a closed lifting $\tilde{w}_{1}$ of $p \tilde{w}$ at $\tilde{e}_{1}$. Therefore, we obtain

$$
p_{*}[\tilde{w}]=[p \tilde{w}]=\left[p \tilde{w}_{1}\right]=p_{*}\left[\tilde{w}_{1}\right] .
$$

Finally, we clearly obtain $p_{*} \pi^{k_{0}}\left(E, e_{0}\right) \subset p_{*} \pi^{k_{0}}\left(E, e_{1}\right)$ and $p_{*} \pi^{k_{0}}\left(E, e_{1}\right) \subset$ $p_{*} \pi^{k_{0}}\left(E, e_{0}\right)$, where $p\left(e_{0}\right)=p\left(e_{1}\right)=b_{0}$.

Conversely, the hypothesis of $p_{*} \pi^{k_{0}}\left(E, e_{0}\right)=p_{*} \pi^{k_{0}}\left(E, e_{1}\right)$ with $p\left(e_{0}\right)=$ $p\left(e_{1}\right)=b_{0}$ implies that $p_{*} \pi^{k_{0}}\left(E, e_{0}\right)$ and $p_{*} \pi^{k_{0}}\left(E, e_{1}\right)$ are conjugates in $\pi^{k_{1}}\left(B, b_{0}\right)$, which makes the map $p$ regular [29].

Corollary 3.14. Let $\left(\left(E, e_{0}\right), p,\left(B, b_{0}\right)\right)$ be a pointed radius $2-\left(k_{0}, k_{1}\right)$ covering and regular and $\left(E, k_{0}\right) k_{0}$-connected. Then $A u t(E \mid B) \simeq \pi^{k_{1}}\left(B, b_{0}\right)$ $/ p_{*} \pi^{k_{0}}\left(E, e_{0}\right)$. 


\section{Concluding Remark and Further Work}

Even though a digital covering space is motivated by a covering space in algebraic topology, a regular covering space in digital covering theory has some intrinsic features. All results have started that given covering maps are required to be radius $2-\left(k_{0}, k_{1}\right)$-covering maps because this property is strongly related to Lemma 3.3. Thus, in case a given covering map $p$ is not a radius $2-\left(k_{0}, k_{1}\right)$-covering map, the existence problem of a regular covering still remains to be studied. In relation to the automorphism group of a digital covering which cannot satisfy a $2-\left(k_{0}, k_{1}\right)$-isomorphism, we can suggest the following open problem.

[Open problem] For a digital covering which cannot satisfy a $2-$ $\left(k_{0}, k_{1}\right)$-isomorphism, how can we calculate its automorphism group?

Furthermore, by using some properties of various types of homeomorphisms and a function space in computer topology [15, 24], we can study computer topological analog of the results in this paper.

\section{References}

[1] L. Boxer, A classical construction for the digital fundamental group, Jour. of Mathematical Imaging and Vision, 10(1999) 51-62.

[2] L. Boxer, Digital Products, Wedge; and Covering Spaces, Jour. of Mathematical Imaging and Vision 25(2006) 159-171.

[3] L. Boxer and I. Karaca, The classification of digital covering spaces, Jour. of Mathematical Imaging and Vision 32(2008) 23-29.

[4] S.E. Han, Computer topology and its applications, Honam Math. Jour. 25(1)(2003) 153-162.

[5] S.E. Han, Algorithm for discriminating digital images w.r.t. a digital $\left(k_{0}, k_{1}\right)$ homeomorphism, Jour. of Applied Mathematics and Computing 18(1-2)(2005) 505-512.

[6] S.E. Han, Digital coverings and their applications, Jour. of Applied Mathematics and Computing 18(1-2)(2005) 487-495.

[7] S.E. Han, Non-product property of the digital fundamental group, Information Sciences 171 (1-3)(2005) 73-91.

[8] S.E. Han, On the simplicial complex stemmed from a digital graph, Honam Mathematical Journal 27 (1),(2005) 115-129.

[9] S.E. Han, Connected sum of digital closed surfaces, Information Sciences 176(3)(2006)332-348.

[10] S.E. Han, Discrete Homotopy of a Closed $k$-Surface, LNCS 4040, SpringerVerlag, Berlin, pp.214-225 (2006).

[11] S.E. Han, Minimal simple closed 18-surfaces and a topological preservation of 3D surfaces, Information Sciences 176(2)(2006) 120-134.

[12] S.E. Han, The fundamental group of a closed $k$-surface, Information Sciences 177(18)(2007) 3731-3748. 
[13] S.E. Han, Strong $k$-deformation retract and its applications, Journal of the Korean Mathematical Society 44(6)(2007) 1479-1503.

[14] S.E. Han, Comparison among digital fundamental groups and its applications, Information Sciences 178(2008) 2091-2104.

[15] S.E. Han, Continuities and homeomorphisms in computer topology, Journal of the Korean Mathematical Society 45(4)(2008) 923-952.

[16] S.E. Han, Equivalent $\left(k_{0}, k_{1}\right)$-covering and generalized digital lifting, Information Sciences 178(2)(2008)550-561.

[17] S.E. Han, Map preserving local properties of a digital image, Acta Applicandae Mathematicae 104(2) (2008) 177-190.

[18] S.E. Han, The $k$-homotopic thinning and a torus-like digital image in $\mathbf{Z}^{n}$, Journal of Mathematical Imaging and Vision 31 (1)(2008) 1-16.

[19] S.E. Han, Cartesian product of the universal covering property, Acta Applicandae Mathematicae (2009) doi 10.1007/s 10440-008-9316-1, Online first publication.

[20] S.E. Han, Existence problem of a generalized universal covering space, Acta Applicandae Mathematicae(2009) doi 10.1007/s 10440-008-9347-7, Online first publication.

[21] S.E. Han, Multiplicative property of the digital fundamental group, Acta Applicandae Mathematicae(2009) doi 10.1007/s 10440-009-9486-5, Online first publication.

[22] S.E. Han, KD- $\left(k_{0}, k_{1}\right)$-homotopy equivalence and its applications, Journal of Korean Mathematical Society, to appear.

[23] S.E. Han, Automorphism group of a cartesian product of digital coverings, Annals of Mathematics, submitted.

[24] S.E. Han, N.D. Georgiou, On computer topological function space Journal of the Korean Mathematical Society 46(4) 841-857 (2009).

[25] E. Khalimsky, Motion, deformation, and homotopy in finite spaces, Proceedings IEEE International Conferences on Systems, Man, and Cybernetics(1987) 227234.

[26] In-Soo Kim, S.E. Han, Digital covering therory and its applications, Honam Mathematical Journal 30(4) (2008) 589-602.

[27] In-Soo Kim, S.E. Han, C.J. Yoo, The pasting property of digital continuity, Acta Applicandae Mathematicae (2009), doi 10.1007/s 10440-008-9422-0, Online first publication.

[28] T.Y. Kong, A. Rosenfeld, Topological Algorithms for the Digital Image Processing, Elsevier Science, Amsterdam, (1996).

[29] W.S. Massey, Algebraic Topology, Springer-Verlag, New York, 1977.

[30] A. Rosenfeld, Digital topology, Am. Math. Mon. 86(1979) 76-87.

[31] A. Rosenfeld and R. Klette, Digital geometry, Information Sciences 148(2003)123-127.

[32] E.H. Spanier, Algebraic Topology, McGraw-Hill Inc., New York, 1966. 
Sang-Eon Han

Faculty of Liberal Education,

Institute of Pure and Applied Mathematics,

Chonbuk National University,

Jeonju-City Jeonbuk, 561-756, Republic of Korea

Tel: +82-63-270-4449

E-mail: sehan@chonbuk.ac.kr 